\title{
SECOND-ORDER FUNCTIONAL PROBLEMS WITH A RESONANCE OF DIMENSION ONE
}

\author{
Nickolai Kosmatov AND WEIHUA JiAnG
}

Abstract. We obtain, using the coincidence degree theory, solvability conditions for all possible resonance scenarios $L u=u^{\prime \prime}=f\left(t, u, u^{\prime}\right)=N u$, with linear functional conditions $B_{i} u=0$, $i=1,2$ with $\operatorname{dim} \operatorname{ker} L=1$. Our work generalizes and improves the results of Zhao and Liang [18] and Cui [3] in several directions. We also construct a meaningful example of a nonlinear functional problem for a pendulum equation which not only satisfies the assumptions of an existence theorem but also has a closed-form solution.

Mathematics subject classification (2010): 34B10, 34B15.

Keywords and phrases: Carathéodory conditions, coincidence degree theory, functional condition, pendulum equation, resonance.

\section{REFERENCES}

[1] A. BuICÁ, Contributions to coincidence degree theory of asymptotically homogeneous operators, Nonlinear. Anal. 68, (2008), 1603-1610.

[2] S. K. Chang AND M. PeI, Solvability for some higher order multi-point boundary value problems at resonance, Results Math. 63, (2013), 763-777.

[3] Y. CUI, Solvability of second-order boundary-value problems at resonance involving integral conditions, Electronic J. Differential Equations 2012, 45 (2012), 1-9.

[4] Z. DU, X. LIN, AND W. GE, A note on a third-order multi-point boundary value problem at resonance, J. Math. Anal. Appl. 302, (2005), 217-229.

[5] M. García-Huidobro, C. P. Gupta, And R. Manásevich, Solvability for a nonlinear threepoint boundary value problem with $p$-Laplacian-like operator at resonance, Abstr. Appl. Anal. 6, 4 (2001), 191-213.

[6] C. P. GuPTA, A second order m-point boundary value problem at resonance Nonlinear Anal. 24, (1995), 1483-1489.

[7] H. Hanche-Olsen And H. Helge, The Kolmogorov-Riesz compactness theorem, Expo. Math. 28, (2010), 385-394.

[8] W. JIANG, Solvability for a coupled system of fractional differential equations at resonance, Nonlinear Anal. Real World Appl. 13, (2012), 2285-2292.

[9] W. JIANG, Solvability of fractional differential equations with p-Laplacian at resonance, Appl. Math. Comput. 260, (2015), 48-56.

[10] W. KARPIŃSKA, On bounded solutions of nonlinear differential equations at resonance, Nonlinear Anal. 51, (2002), 723-733.

[11] E. R. KaUfmann, A third order non-local boundary value problem at resonance, Electron. J. Qual. Theory Differ. Equ., Spec. Ed. 1, (2009), 1-11.

[12] N. Kosmatov, A multi-point boundary value problem with two critical conditions, Nonlinear Anal. 65, 3 (2006), 622-633.

[13] D. Maroncelli, Scalar multi-point boundary value problems at resonance, Differ. Equ. Appl. 7, (2015), 449-468.

[14] J. MAWHIn, Topological degree methods in nonlinear boundary value problems, NSF-CBMS Regional conference Series in Math. 40, Amer. Math. Soc., Providence, RI, 1979. 
[15] M. Mohamed, B. Thompson, And M. S. Jusoh, First-order three-point BVPs at resonance (II), Electron. J. Qual. Theory Differ. Equ. 2011, 68 (2011), 1-21.

[16] P.D. Phung AND L.X. TRuong, On the existence of a three point boundary value problem at resonance in $\mathbb{R}^{n}$, J. Math. Anal. Appl. 416, (2014), 522-533.

[17] A. J. YANG, An extension of Leggett-Williams norm-type theorem for coincidences and its application, Topol. Methods Nonlinear Anal. 37, 1 (2011), 177-191.

[18] Z. ZHAO AND J. LIANG, Existence of solutions to functional boundary value problem of second-order nonlinear differential equation, J. Math. Anal. Appl. 373, (2011), 614-634. 